\title{
Newton's law of heating and the heat equation
}

\author{
Mark Gockenbach and Kristin Schmidtke
}

(Communicated by Suzanne Lenhart)

\begin{abstract}
Newton's law of heating models the average temperature in an object by a simple ordinary differential equation, while the heat equation is a partial differential equation that models the temperature as a function of both space and time. A series solution of the heat equation, in the case of a spherical body, shows that Newton's law gives an accurate approximation to the average temperature if the body is not too large and it conducts heat much faster than it gains heat from the surroundings. Finite element simulation confirms and extends the analysis.
\end{abstract}

\section{Introduction}

A popular application involving an elementary differential equation is Newton's law of heating, which describes the change in temperature in an object whose surroundings are hotter than it is. ${ }^{1}$ If the temperature at time $t$ is $T(t)$, then Newton's law of heating is

$$
T^{\prime}=\bar{\alpha}\left(T_{s}-T\right), \quad T(0)=T_{0},
$$

where $T_{s}$ and $T_{0}$ are constants representing the temperature of the surroundings and the initial temperature of the object, respectively. ${ }^{2}$ The differential equation in (1) simply states that the rate of change of the temperature is proportional to the difference between the temperatures of the surroundings and the object. The solution of (1) is

$$
T(t)=T_{s}-\left(T_{s}-T_{0}\right) e^{-\bar{\alpha} t}
$$

MSC2000: 35K05.

Keywords: heat equation, Newton's law of heating, finite elements, Bessel functions.

${ }^{1}$ If the surroundings are colder, then the differential equation is called Newton's law of cooling. For definiteness of language, we will usually assume that heating is occurring.

${ }^{2}$ We take $T_{0}$ and $T_{S}$ to be constants, as this agrees with the usual textbook presentation of Newton's law of heating, which we wish to analyze in this paper. There is nothing that would prevent us from allowing $T_{s}$ to depend on time, or $T_{0}$ to depend on space (so that $T_{0}(x, y, z)$ is the initial temperature at the point $(x, y, z)$ in the object). If $T_{0}$ were variable, then we would use the average value $\bar{T}_{0}$ of $T_{0}$ in the ordinary differential equation model (1) and the variable $T_{0}$ itself in the partial differential equation model presented below. Allowing nonconstant $T_{0}$ and/or $T_{S}$ would considerably complicate the analysis in this paper. 
Newton's law of heating is included in virtually every introductory textbook on differential equations, such as [Boyce and DiPrima 1992; Zill 2005].

Newton's law of heating assumes that the temperature of the object is represented by a single number. A more sophisticated model represents the object as occupying a domain $\Omega$ in $\mathrm{R}^{3}$ and its temperature as a function $u(x, y, z, t)$, where $(x, y, z) \in \Omega$. The governing partial differential equation is the heat equation

$$
\rho c \frac{\partial u}{\partial t}=\kappa \Delta u \text { in } \Omega, \quad t>0 .
$$

In this equation, $\Delta u$ is the Laplacian of $u$ :

$$
\Delta u=\frac{\partial^{2} u}{\partial x^{2}}+\frac{\partial^{2} u}{\partial y^{2}}+\frac{\partial^{2} u}{\partial z^{2}} .
$$

The quantities $\rho, c$, and $\kappa$ describe the material properties of the object; $\rho$ is the density, $c$ is the specific heat, and $\kappa$ is the thermal conductivity. Typically, $\kappa$ is given in $\mathrm{J} / \mathrm{s} \mathrm{cm} \mathrm{K,} \rho$ in $\mathrm{g} / \mathrm{cm}^{3}$, and $c$ in $\mathrm{J} / \mathrm{g} \mathrm{K}$. For a derivation of the heat equation, the reader can consult introductory books on partial differential equations, such as [Gockenbach 2002; Haberman 2004].

To obtain a complete description of $u$, we must model how $\Omega$ exchanges heat energy with its surroundings. We adopt a model much like Newton's law of heating:

$$
\kappa \frac{\partial u}{\partial n}=\alpha\left(T_{s}-u\right) \quad \text { on } \partial \Omega .
$$

This is called a Robin boundary condition; it states that the heat flux across the boundary is proportional to the difference between $T_{s}$ and the temperature on $\partial \Omega$. Although the form of the boundary condition is analogous to Newton's law of heating, there is no reason to expect that the constants $\bar{\alpha}$ and $\alpha$ are the same; indeed, since they have different units, it would be surprising if their numerical values were the same.

The PDE (3) and the boundary condition (4), together with an initial condition, form a well-posed problem that determines $u$ uniquely. We assume that the initial temperature in $\Omega$ is constant and obtain the following initial boundary value problem (IBVP):

$$
\begin{aligned}
\rho c \frac{\partial u}{\partial t}-\kappa \Delta u=0 & \text { in } \Omega, t>0, \\
u(x, y, z, 0)=T_{0} & \text { in } \Omega, \\
\kappa \frac{\partial u}{\partial n}+\alpha u=\alpha T_{s} & \text { on } \partial \Omega, t>0 .
\end{aligned}
$$

We now have two models to describe the temperature of the given object, namely, Newton's law of heating and the heat equation together with a Robin boundary 
condition. The simpler model (1) would be an adequate substitute for the more complicated model (5) if $T$ is close to the average temperature in $\Omega$ as predicted by (5):

$$
\bar{u}=\frac{1}{|\Omega|} \int_{\Omega} u .
$$

Before we can compare the solutions $T$ and $\bar{u}$, we must determine the relative values of the constants $\bar{\alpha}$ and $\alpha$ appearing in (1) and (5), respectively. Fortunately, given $\alpha$, the value of $\bar{\alpha}$ is suggested by the following calculation:

$$
\begin{aligned}
\frac{d \bar{u}}{d t}=\frac{1}{|\Omega|} \int_{\Omega} \frac{\partial u}{\partial t} & =\frac{1}{\rho c|\Omega|} \int_{\Omega} \rho c \frac{\partial u}{\partial t}=\frac{1}{\rho c|\Omega|} \int_{\Omega} \kappa \Delta u \\
& =\frac{1}{\rho c|\Omega|} \int_{\partial \Omega} \kappa \frac{\partial u}{\partial n}=\frac{1}{\rho c|\Omega|} \int_{\partial \Omega} \alpha\left(T_{S}-u\right) .
\end{aligned}
$$

If we define $\bar{u}_{b}=\bar{u}_{b}(t)$ to be the average value of $u$ on $\partial \Omega$,

$$
\bar{u}_{b}=\frac{1}{|\partial \Omega|} \int_{\partial \Omega} u
$$

then

$$
\int_{\partial \Omega} \alpha\left(T_{s}-u\right)=\alpha|\partial \Omega|\left(T_{s}-\bar{u}_{b}\right)
$$

and we obtain

$$
\bar{u}^{\prime}=\frac{\alpha|\partial \Omega|}{\rho c|\Omega|}\left(T_{s}-\bar{u}_{b}\right) .
$$

We then see that $\bar{u}$ satisfies a differential equation similar to Newton's law of heating, but with $\alpha$ replaced with

$$
\bar{\alpha}=\frac{|\partial \Omega|}{\rho c|\Omega|} \alpha
$$

Of course, even with this value of $\bar{\alpha},(1)$ and (7) are not the same, since (7) shows that the rate of change of $\bar{u}$ is determined not by $\bar{u}$ itself, but by $\bar{u}_{b}$. However, the equations are similar enough that we might expect $T$ to be a good approximation to $\bar{u}$.

The primary purpose of this paper is to compare the solutions of the heat equation (5) and Newton's law of heating (1), where $\bar{\alpha}$ is given by (8). We will use both analytical and numerical methods; to make the analysis tractable and the numerics simpler, we will assume that the object is spherical. 


\section{Solution of the heat equation on a spherical domain}

We will henceforth assume that $\Omega$ is the ball of radius $R$ centered at the origin. Since the initial value of $u$ is a constant and the boundary conditions are constant on $\partial \Omega$, the solution to the IBVP (5) depends only on the radial variable $r=\sqrt{x^{2}+y^{2}+z^{2}}$. This follows from the fact that the Laplacian $\Delta$ is invariant under any rotation of the coordinate system (for a nice discussion of this, the reader can consult [Folland 1995, Section 2.A]). We can therefore write $u=u(r, t)$, and we recall that

$$
\Delta u=\frac{\partial^{2} u}{\partial r^{2}}+\frac{2}{r} \frac{\partial u}{\partial r}
$$

(see, for example, [Haberman 2004]). The IBVP (5) can thus be rewritten as

$$
\begin{aligned}
\rho c \frac{\partial u}{\partial t}-\kappa\left(\frac{\partial^{2} u}{\partial r^{2}}+\frac{2}{r} \frac{\partial u}{\partial r}\right) & =0, & & 0<r<R, t>0, \\
u(r, 0) & =T_{0}, & & 0<r<R, \\
\kappa \frac{\partial u}{\partial r}(R, t)+\alpha u(R, t) & =\alpha T_{s}, & & t>0 .
\end{aligned}
$$

In addition to the equations explicitly listed above, there is the implicit requirement that $u$ be finite at $r=0$. We use the technique of shifting the data to transform (9) to a problem with homogeneous boundary conditions. We define $Y(r)=a r^{2}$, where $a$ is chosen so that $Y$ satisfies the boundary condition $\kappa Y^{\prime}(R)+\alpha Y(R)=\alpha T_{s}$,

$$
a=\frac{\alpha T_{s}}{2 \kappa R+\alpha R^{2}},
$$

and write $u(r, t)=U(r, t)+Y(r)$. Then $U$ satisfies

$$
\begin{aligned}
\rho c \frac{\partial U}{\partial t}-\kappa\left(\frac{\partial^{2} U}{\partial r^{2}}+\frac{2}{r} \frac{\partial U}{\partial r}\right) & =6 a \kappa, & & 0<r<R, t>0, \\
U(r, 0) & =T_{0}-a r^{2}, & & 0<r<R, \\
\kappa \frac{\partial U}{\partial r}(R, t)+\alpha U(R, t) & =0, & & t>0 .
\end{aligned}
$$

We can derive a solution to (11) by expanding $U$ in terms of the eigenfunctions of the spatial operator

$$
L=-\kappa\left(\frac{d^{2}}{d r^{2}}+\frac{2}{r} \frac{d}{d r}\right),
$$

where homogeneous Robin conditions are imposed on the eigenfunctions:

$$
\kappa v^{\prime}(R)+\alpha v(R)=0 .
$$


We will now briefly derive these eigenfunctions and the corresponding eigenvalues. First, using integration by parts, we can show that if $v_{1}, v_{2}$ satisfy (12), then

$$
\int_{0}^{R}-\kappa\left(\frac{d^{2} v_{1}}{d r^{2}}+\frac{2}{r} \frac{d v_{1}}{d r}\right) v_{2}(r) r^{2} d r=\alpha v_{1}(R) v_{2}(R) R^{2}+\int_{0}^{R} \kappa \frac{d v_{1}}{d r} \frac{d v_{2}}{d r} r^{2} d r .
$$

This shows that $L$ is symmetric with respect to the inner product

$$
\left\langle v_{1}, v_{2}\right\rangle=\int_{0}^{r} v_{1}(r) v_{2}(r) r^{2} d r,
$$

that is, that $\left\langle L\left(v_{1}\right), v_{2}\right\rangle=\left\langle v_{1}, L\left(v_{2}\right)\right\rangle$ for all $v_{1}, v_{2}$ satisfying the given boundary conditions. The standard argument then shows that the eigenvalues of $L$ are all real, and that eigenfunctions of $L$ corresponding to distinct eigenvalues are orthogonal with respect to the given inner product [Gockenbach 2002, Section 5.1]. We also see that

$$
\langle L(v), v\rangle=\alpha v(R)^{2}+\int_{0}^{R} \kappa\left(\frac{d v}{d r}(r)\right)^{2} r^{2} d r
$$

which is positive for every nonzero function $v$. This implies that all the eigenvalues of $L$ are positive.

We now wish to solve

$$
\begin{aligned}
-\kappa\left(\frac{d^{2} v}{d r^{2}}+\frac{2}{r} \frac{d v}{d r}\right) & =\lambda v, \quad 0<r<R, \\
\kappa v^{\prime}(R)+\alpha v(R) & =0,
\end{aligned}
$$

for $\lambda>0$ and $v=v(r)$. It is well known [Arfken and Weber 2005] that the only solutions to $(13)_{1}$ that are bounded at the origin are multiples of

$$
j_{0}(\sqrt{\lambda / \kappa} r)
$$

where $j_{0}$ is the first spherical Bessel function:

$$
j_{0}(s)=\frac{\sin (s)}{s} .
$$

(For more information about Bessel functions, including the properties cited below, the reader can consult [Trantor 1968] or the comprehensive reference [?].) The problem of finding the eigenvalues and eigenfunctions then reduces to finding the values of $\lambda>0$ such that $v(r)=j_{0}(\sqrt{\lambda / \kappa} r)$ satisfies the boundary condition $(13)_{2}$.

Substituting $v$ into $(13)_{2}$ and simplifying yields

$$
\tan (s)=m s, \quad m=\frac{\kappa}{\kappa-\alpha R}, \quad s=R \sqrt{\lambda / \kappa} .
$$


We will henceforth make the important assumption that $\kappa-\alpha R>0$ or, equivalently, that

$$
\beta=\frac{\alpha R}{\kappa}<1
$$

Recalling that $\kappa$ is the thermal conductivity within the object and $\alpha$ describes how well thermal energy is transmitted to the environment, this assumption means that the object conducts energy more quickly than it transmits energy to the surroundings and also that the radius of the object is not too large. Our intuition ought to tell us that these are precisely the conditions under which Newton's law of heating should give accurate results. In fact, below we will expand $\bar{u}(t)-T(t)$ in powers of $\beta$ and show that $\bar{u}(t)-T(t)=O(\beta)$ uniformly for $t \geq 0$.

Assumption (15) implies that $m=1 /(1-\beta)>1$ in (14), and a simple graph then shows that (14) has positive solutions $s_{1}, s_{2}, s_{3}, \ldots$, with

$$
(k-1) \pi<s_{k}<\left(k-\frac{1}{2}\right) \pi, k=1,2,3, \ldots
$$

and $s_{k} \approx\left(k-\frac{1}{2}\right) \pi$ for $k \geq 2$. For our analysis below, we need accurate estimates of the $s_{k}$ 's. To estimate $s_{1}$, we can expand $\tan (s)$ in powers of $s$, truncate the series, and obtain

$$
\begin{aligned}
& s_{1}=\sqrt{3} \beta^{1 / 2}-\frac{\sqrt{3}}{10} \beta^{3 / 2}+O\left(\beta^{5 / 2}\right), \\
& \lambda_{1}=\frac{3 \kappa}{R^{2}}\left(\beta-\frac{1}{5} \beta^{2}+O\left(\beta^{3}\right)\right) .
\end{aligned}
$$

For $k \geq 2$, we see that each $s_{k}$ is greater than the corresponding solution $\bar{s}_{k}$ to $\tan (s)=s$. We write $\bar{s}_{k}=\left(k-\frac{1}{2}\right) \pi-\epsilon_{k}$, expand $\tan \left(\left(k-\frac{1}{2}\right) \pi-\epsilon_{k}\right)$ in powers of $\epsilon_{k}$, and solve to get

$$
s_{k} \geq \bar{s}_{k}=\left(k-\frac{1}{2}\right) \pi\left(1-\frac{1}{\left(k-\frac{1}{2}\right)^{2} \pi^{2}}-\frac{2}{3\left(k-\frac{1}{2}\right)^{4} \pi^{4}}+\ldots\right) .
$$

In particular, we find that

$$
0.95\left(k-\frac{1}{2}\right) \pi<s_{k}<\left(k-\frac{1}{2}\right) \pi, \quad k=2,3, \ldots
$$

This estimate will suffice for our purposes below.

We now write

$$
v_{k}(r)=j_{0}\left(\sqrt{\lambda_{k} / \kappa} r\right)=\frac{\sin \left(\sqrt{\lambda_{k} / \kappa} r\right)}{\sqrt{\lambda_{k} / \kappa} r}, \quad k=1,2,3, \ldots,
$$

for the eigenfunctions of $L$. The standard theory of (spherical) Bessel functions guarantees that any function $u=u(r)$ (finite at the origin) can be expanded in terms 
of the orthogonal functions $v_{1}, v_{2}, v_{3}, \ldots$ We can therefore expand the solution $U$ of (11) as

$$
U(r, t)=\sum_{k=1}^{\infty} C_{k}(t) v_{k}(r) .
$$

Substituting this expression into the PDE (11) $)_{1}$ yields

$$
\sum_{k=1}^{\infty}\left\{\rho c C_{k}^{\prime}(t)+\lambda_{k} C_{k}(t)\right\} v_{k}(r)=\sum_{k=1}^{\infty} b_{k} v_{k}(r),
$$

where $\sum_{k=1}^{\infty} b_{k} v_{k}(r)$ is the expansion of the constant function $6 a \kappa$ :

$$
b_{k}=\frac{6 a \kappa \int_{0}^{R} v_{k}(r) r^{2} d r}{\int_{0}^{R} v_{k}(r)^{2} r^{2} d r}, \quad k=1,2,3, \ldots
$$

From the initial condition $(11)_{2}$, we have

$$
\sum_{k=1}^{\infty} C_{k}(0) v_{k}(r)=\sum_{k=1}^{\infty} d_{k} v_{k}(r),
$$

where $\sum_{k=1}^{\infty} d_{k} v_{k}(r)$ is the expansion of $T_{0}-a r^{2}$ :

$$
d_{k}=\frac{\int_{0}^{R}\left(T_{0}-a r^{2}\right) v_{k}(r) r^{2} d r}{\int_{0}^{R} v_{k}(r)^{2} r^{2} d r}, k=1,2,3, \ldots
$$

We then obtain the following initial value problem for $C_{k}$ :

$$
\rho c C_{k}^{\prime}+\lambda_{k} C_{k}=b_{k}, C_{k}(0)=d_{k}, \quad k=1,2,3, \ldots
$$

The solution is

$$
C_{k}(t)=\frac{b_{k}}{\lambda_{k}}+\left(d_{k}-\frac{b_{k}}{\lambda_{k}}\right) e^{-\lambda_{k} t /(\rho c)}, \quad k=1,2,3, \ldots
$$

We now have the following solution to the IBVP (9):

$$
u(r, t)=a r^{2}+\sum_{k=1}^{\infty} C_{k}(t) v_{k}(r) .
$$

The reader will recall that

$$
\bar{u}=\frac{1}{|\Omega|} \int_{\Omega} u .
$$

With $\Omega$ equal to the ball of radius $R$, this reduces to

$$
\bar{u}(t)=\frac{4 \pi}{(4 / 3) \pi R^{3}} \int_{0}^{R} u(r, t) r^{2} d r=\frac{3}{R^{3}} \int_{0}^{R} u(r, t) r^{2} d r,
$$


and so

$$
\bar{u}(t)=\frac{3}{R^{3}} \int_{0}^{R} a r^{4} d r+\sum_{k=1}^{\infty}\left\{\frac{3}{R^{3}} \int_{0}^{R} v_{k}(r) r^{2} d r\right\} C_{k}(t)
$$

(since the solution $u$ to the heat equation is known to be smooth, the series for $u$ can be integrated term-by-term to produce (24)).

\section{Comparing the solutions}

We now have formulas for both $T(t)$ and $\bar{u}(t)$, and we wish to bound $|T(t)-\bar{u}(t)|$ for $t \geq 0$. Since $v_{k}$ is oscillatory for $k \geq 2$ (see Figure 1 and also the definition of $v_{k}$ in (19)), we expect

$$
\frac{3}{R^{3}} \int_{0}^{R} v_{k}(r) r^{2} d r
$$

to be small for $k \geq 2$. We hypothesize, then, that $\bar{u}(t)$ will be well approximated by

$$
\bar{u}_{1}(t)=\frac{3}{R^{3}} \int_{0}^{R} a r^{4} d r+\left(\frac{3}{R^{3}} \int_{0}^{R} v_{1}(r) r^{2} d r\right) C_{1}(t) .
$$

We therefore wish to show that $\left|T(t)-\bar{u}_{1}(t)\right|$ and $\left|\bar{u}_{1}(t)-\bar{u}(t)\right|$ are both small for $t \geq 0$.

A straightforward calculation shows that

$$
\frac{3}{R^{3}} \int_{0}^{R} v_{k}(r) r^{2} d r=3 \frac{\sin \left(R \sqrt{\lambda_{k} / \kappa}\right)-R \sqrt{\lambda_{k} / \kappa} \cos \left(R \sqrt{\lambda_{k} / \kappa}\right)}{\left(R \sqrt{\lambda_{k} / \kappa}\right)^{3}},
$$

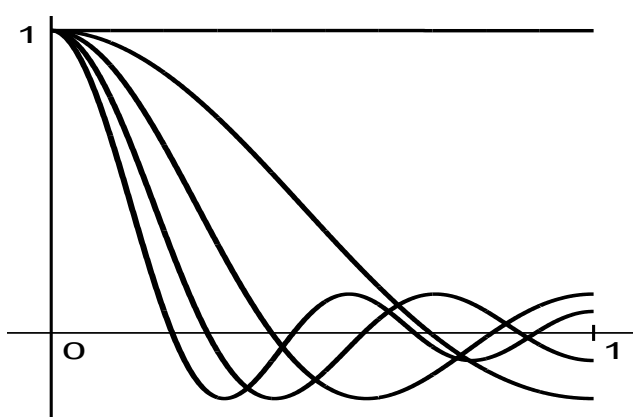

Figure 1. The first five eigenfunctions $v_{1}, v_{2}, v_{3}, v_{4}, v_{5}$ on the interval $[0, R], R=1.0$. To construct this graph, we have taken $\kappa=1.0$ and $\alpha=0.001$. The first eigenfunction is nearly constant on the interval $[0, R]$, while, for $k \geq 2, v_{k}$ is increasingly oscillatory as $k$ increases. 
or

$$
\frac{3}{R^{3}} \int_{0}^{R} v_{k}(r) r^{2} d r=3 \frac{\sin \left(s_{k}\right)-s_{k} \cos \left(s_{k}\right)}{s_{k}^{3}} .
$$

Since $\tan \left(s_{k}\right)=m s_{k}$, or $s_{k} \cos s_{k}=m^{-1} \sin s_{k}$, we obtain

$$
\frac{3}{R^{3}} \int_{0}^{R} v_{k}(r) r^{2} d r=3 \frac{m-1}{m} \frac{\sin s_{k}}{s_{k}^{3}}=3 \beta \frac{\sin s_{k}}{s_{k}^{3}} .
$$

For $k \geq 2$, we can apply (18) to obtain

$$
\left|\frac{3}{R^{3}} \int_{0}^{R} v_{k}(r) r^{2} d r\right| \leq \frac{3 \beta}{s_{k}^{3}} \leq \frac{3 \beta}{(0.95(k-1 / 2) \pi)^{3}},
$$

which yields

$$
\left|\frac{3}{R^{3}} \int_{0}^{R} v_{k}(r) r^{2} d r\right| \leq \frac{1}{8(k-1 / 2)^{3}} \beta, \quad k=2,3, \ldots
$$

For $k=1$, we expand the integral in powers of $\beta$, which is a straightforward calculation: ${ }^{3}$

$$
\frac{3}{R^{3}} \int_{0}^{R} v_{1}(r) r^{2} d r=1-\frac{3}{10} \beta+O\left(\beta^{2}\right) .
$$

The results given in (25) and (27) support our hypothesis that $\bar{u}(t)$ should be well approximated by $\bar{u}_{1}(t)$.

We can now complete the bound $\left|T(t)-\bar{u}_{1}(t)\right|$ in short order. From $(16)_{2},(20)$, and (21), we find that

$$
\frac{b_{1}}{\lambda_{1}}=T_{s}+O\left(\beta^{2}\right)
$$

and

$$
d_{1}-\frac{b_{1}}{\lambda_{1}}=T_{0}-T_{s}+\frac{3}{10}\left(T_{0}-T_{s}\right) \beta+O\left(\beta^{2}\right) .
$$

We can also expand the constant term in the series for $\bar{u}(t)$ in powers of $\beta$ :

$$
\frac{3}{R^{3}} \int_{0}^{R} a r^{4} d r=\frac{3 a R^{2}}{5}=\frac{3 T_{s}}{10} \beta+O\left(\beta^{2}\right) .
$$

Putting these results together, we obtain

$$
\bar{u}_{1}(t)=T_{s}-\left(T_{s}-T_{0}\right) e^{-\lambda_{1} t /(\rho c)}+O\left(\beta^{2}\right) .
$$

The reader should notice how the $O(\beta)$ term has canceled (compare the product of (27) and (28)). The only dependence of the $O\left(\beta^{2}\right)$ term on $t$ is through the exponential (this dependence is not shown explicitly here), which is bounded by

\footnotetext{
${ }^{3}$ We used Mathematica to generate this and other series expansions.
} 
one for $t \geq 0$. Thus the $O\left(\beta^{2}\right)$ term is uniformly small for $t \geq 0$ (assuming $\beta$ is small).

The similarity between $T(t)$ and $\bar{u}_{1}(t)$ is now obvious (compare (2) and (29)). It remains only to compare the exponentials $e^{-\lambda_{1} t /(\rho c)}$ and $e^{-\bar{\alpha} t}=e^{-3 \alpha t /(\rho c R)}$. We have

$$
\lambda_{1}=\frac{3 \kappa}{R^{2}} \beta\left(1-\frac{1}{5} \beta+O\left(\beta^{2}\right)\right)=\frac{3 \alpha}{R}\left(1-\frac{1}{5} \beta+O\left(\beta^{2}\right)\right)
$$

(using $\beta=\alpha R / \kappa)$, so we see that $\lambda_{1} /(\rho c)$ and $\bar{\alpha}=3 \alpha /(\rho c R)$ are quite similar, with

$$
e^{-\lambda_{1} t /(\rho c)} \geq e^{-\bar{\alpha} t}, \quad t \geq 0 .
$$

To obtain a useful bound, we maximize the function

$$
f(t)=e^{-\lambda_{1} t /(\rho c)}-e^{-\bar{\alpha} t}, \quad t \geq 0 .
$$

The function $f$ has a unique stationary point, and we easily obtain

$$
0 \leq e^{-\lambda_{1} t /(\rho c)}-e^{-\bar{\alpha} t} \leq \frac{1}{5 e} \beta+O\left(\beta^{2}\right), \quad t \geq 0 .
$$

We can now bound the difference between $T(t)$ and $\bar{u}_{1}(t)$ :

$$
\begin{aligned}
\left|T(t)-\bar{u}_{1}(t)\right| & =\left|T_{s}-\left(T_{s}-T_{0}\right) e^{-\bar{\alpha} t}-T_{s}+\left(T_{s}-T_{0}\right) e^{-\lambda_{1} t /(\rho c)}+O\left(\beta^{2}\right)\right| \\
& =\left|\left(T_{s}-T_{0}\right)\left(e^{-\lambda_{1} t /(\rho c)}-e^{-\bar{\alpha} t}\right)\right|+O\left(\beta^{2}\right) \\
& \leq \frac{\left|T_{s}-T_{0}\right|}{5 e} \beta+O\left(\beta^{2}\right) .
\end{aligned}
$$

As noted above, this bound is uniform over the interval $0 \leq t<\infty$.

Finally, we bound $\left|\bar{u}_{1}(t)-\bar{u}(t)\right|$ for $t \geq 0$. We will merely sketch the results, which the interested reader can verify. We already have the upper bound (26) for

$$
\left|\frac{3}{R^{3}} \int_{0}^{R} v_{k}(r) r^{2} d r\right| \text {. }
$$

We will need upper bounds for $d_{k}$ and $b_{k} / \lambda_{k}$, which will require a lower bound for

$$
\int_{0}^{R} v_{k}(r)^{2} r^{2} d r
$$

A straightforward calculation gives

$$
\int_{0}^{R} v_{k}(r)^{2} r^{2} d r=\frac{\kappa}{\lambda_{k}}\left(\frac{R}{2}-\frac{\sin \left(2 \sqrt{\lambda_{k} / \kappa} R\right)}{4 \sqrt{\lambda_{k} / \kappa}}\right) \geq \frac{R^{3}}{4(k-1 / 2)^{2} \pi^{2}}
$$

(applying the upper bound for $\lambda_{k}$ implied by (18)). We then have

$$
b_{k}=\frac{6 a \kappa \int_{0}^{R} v_{k}(r) r^{2} d r}{\int_{0}^{R} v_{k}(r)^{2} r^{2} d r}
$$


and (26) gives an upper bound for the numerator. Applying this upper bound together with (32) and simplifying yields

$$
b_{k} \leq \frac{3 \kappa T_{s} \pi^{2}}{2 R^{5}\left(k-\frac{1}{2}\right)} \beta^{2} .
$$

Since (18) implies

$$
\lambda_{k} \geq 0.95^{2}\left(k-\frac{1}{2}\right)^{2} \pi^{2} \kappa, \quad k=2,3, \ldots,
$$

we obtain (after a little manipulation)

$$
\frac{b_{k}}{\lambda_{k}} \leq \frac{2 T_{s}}{R^{5}\left(k-\frac{1}{2}\right)^{3}} \beta^{2} .
$$

Obtaining a bound for $d_{k}$ is more work. We have

$$
\begin{array}{r}
\int_{0}^{R}\left(T_{0}-a r^{2}\right) v_{k}(r) r^{2} d r \\
=\frac{\kappa}{(2+\beta) \lambda_{k}^{5 / 2} R^{2}} \cdot\left\{\begin{array}{r}
\lambda_{k} R\left(6 \beta \kappa T_{s}+\lambda_{k} R^{2}\left((2+\beta) T_{0}-\beta T_{s}\right)\right) \cos \left(\sqrt{\lambda_{k} / \kappa} R\right) \\
\left.\quad+\sqrt{\kappa}\left(6 \beta \kappa T_{s}+\lambda_{k} R^{2}\left((2+\beta) T_{0}-3 \beta T_{s}\right)\right) \sin \left(\sqrt{\lambda_{k} / \kappa} R\right)\right\} \\
=\frac{\kappa^{3 / 2}}{(2+\beta) \lambda_{k}^{5 / 2} R^{2}} \cdot\left\{\left(\sin s_{k}-s_{k} \cos s_{k}\right)\left(6 \beta \kappa T_{s}+\lambda_{k} R^{2}\left((2+\beta) T_{0}-\beta T_{s}\right)\right)\right. \\
\left.-2 \beta T_{2} \sin s_{k}\right\} .
\end{array}\right.
\end{array}
$$

Since $\sin s_{k}=m s_{k} \cos s_{k}$, we have

$$
\sin s_{k}-s_{k} \cos s_{k}=(m-1) s_{k} \cos s_{k}=\frac{\beta}{1-\beta} s_{k} \cos s_{k} .
$$

Also, $s_{k}$ is an approximate root of cosine; using (17) and the Taylor expansion of cosine around $s=(k-1 / 2) \pi$, we obtain $s_{k} \cos s_{k}=O(1)$. Using this and some more manipulation, we find positive constants $\gamma_{1}$ and $\gamma_{2}$ such that

$$
\left|\int_{0}^{R}\left(T_{0}-a r^{2}\right) v_{k}(r) r^{2} d r\right| \leq \frac{\gamma_{1} \beta+\gamma_{2} \beta^{2}}{\lambda_{k}^{3 / 2}}, \quad k=2,3, \ldots
$$

This, together with the lower bound (32), yields

$$
d_{k}=\frac{\int_{0}^{R}\left(T_{0}-a r^{2}\right) v_{k}(r) r^{2} d r}{\int_{0}^{R} v_{k}(r)^{2} r^{2} d r} \leq \bar{\gamma}_{1} \beta+\bar{\gamma}_{2} \beta^{2},
$$

where $\bar{\gamma}_{1}$ and $\bar{\gamma}_{2}$ are positive constants. 
We can finally use (26), (34), and (35) to bound $\left|\bar{u}_{1}(t)-\bar{u}(t)\right|$. We have

$$
\begin{aligned}
\left|\bar{u}_{1}(t)-\bar{u}(t)\right| & \leq \sum_{k=2}^{\infty}\left(\frac{3}{R^{3}} \int_{0}^{R} v_{k}(r) r^{2} d r\right)\left|\frac{b_{k}}{\lambda_{k}}+\left(d_{k}-\frac{b_{k}}{\lambda_{k}}\right) e^{-\lambda_{k} t /(\rho c)}\right| \\
& \leq \sum_{k=2}^{\infty} \frac{\beta}{8(k-1 / 2)^{3}}\left(2\left|\frac{b_{k}}{\lambda_{k}}\right|+\left|d_{k}\right|\right) .
\end{aligned}
$$

Since

$$
\sum_{k=2}^{\infty} \frac{1}{\left(k-\frac{1}{2}\right)^{3}}
$$

is finite, (34) and (35) yield positive constants $\tilde{\gamma}_{1}$ and $\tilde{\gamma}_{2}$ such that

$$
\left|\bar{u}_{1}(t)-\bar{u}(t)\right| \leq \tilde{\gamma}_{1} \beta^{2}+\tilde{\gamma}_{2} \beta^{3}, t \geq 0 .
$$

This, together with our earlier bound on $\left|T(t)-\bar{u}_{1}(t)\right|$, yields our final result:

$$
|T(t)-\bar{u}(t)| \leq \frac{\left|T_{s}-T_{0}\right|}{5 e} \beta+O\left(\beta^{2}\right), \quad t \geq 0 .
$$

The reader will recall that

$$
\beta=\frac{\alpha R}{\kappa},
$$

where $\kappa$ is the thermal conductivity with the object, $\alpha$ describes how well the object transmits heat energy to its surroundings (or vice versa), and $R$ is the radius of $\Omega$. As long as $\alpha \ll \kappa$ and $\Omega$ is not too large, (36) shows that the average temperature in $\Omega$ will be well approximated by Newton's law of heating.

\section{The finite element method}

We wish to give some numerical examples to illustrate the above analysis. This requires that we be able to compute accurate solutions to the initial-boundary value problem (9). We will use the standard Galerkin-Crank-Nicolson finite element method, which we now briefly describe.

To compute the solution of (9), we first rewrite the problem in its variational form:

$$
\begin{array}{r}
\int_{0}^{R} \rho c \frac{\partial u}{\partial t}(r, t) v(r) r^{2} d r+\int_{0}^{R} \kappa \frac{\partial u}{\partial r}(r, t) v^{\prime}(r) r^{2} d r+\alpha R^{2} u(R, t) v(R) \\
=\alpha R^{2} T_{s} v(R), \quad \text { for all } v \in V .
\end{array}
$$

Here $V$ is the space of test functions,

$$
V=\left\{v \in H^{1}(0, R): r v \in L^{2}(0, R), r v^{\prime} \in L^{2}(0, R)\right\},
$$


based on the Sobolev space $H^{1}(0, R)$ (the space of functions with one squareintegrable (weak) derivative). The variational form (37) results from multiplying the PDE (9) by a test function, integrating over $\Omega$, integrating the $\partial^{2} u / \partial r^{2}$ term by parts, and applying the boundary condition. It is well known that the variational form is equivalent to the original initial-boundary value problem (at least when, as in this case, the original problem is known to have a smooth solution).

We obtain the semidiscrete form of (37) by discretizing in space using piecewise linear functions on a mesh defined by $r_{i}=i h, h=R / n$, and applying Galerkin's method. We will write $V_{h}$ for the space of continuous piecewise linear functions on the given mesh, and $\left\{\phi_{0}, \phi_{1}, \ldots, \phi_{n}\right\}$ for the usual nodal basis defined by

$$
\phi_{i}\left(r_{j}\right)= \begin{cases}1 & \text { if } i=j \\ 0 & \text { if } i \neq j\end{cases}
$$

The semidiscrete solution is

$$
u_{h}(r, t)=\sum_{j=0}^{n} \alpha_{j}(t) \phi_{j}(r),
$$

satisfying

$$
\begin{array}{r}
\int_{0}^{R} \rho c \frac{\partial u_{h}}{\partial t}(r, t) v(r) r^{2} d r+\int_{0}^{R} \kappa \frac{\partial u_{h}}{\partial r}(r, t) v^{\prime}(r) r^{2} d r+\alpha R^{2} u_{h}(R, t) v(R) \\
=\alpha R^{2} T_{s} v(R), \quad \text { for all } v \in V_{h} .
\end{array}
$$

Choosing $v=\phi_{i}, i=0,1, \ldots, n,(38)$ is equivalent to

$$
M a^{\prime}+(K+G) a=F,
$$

where $M$ and $K$ are the mass and stiffness ${ }^{4}$ matrices,

$$
M_{i j}=\int_{0}^{R} \rho c \phi_{j}(r) \phi_{i}(r) r^{2} d r, K_{i j}=\int_{0}^{R} \kappa \phi_{j}^{\prime}(r) \phi_{i}^{\prime}(r) r^{2} d r, \quad i, j=0,1, \ldots, n .
$$

Every entry in the matrix $G$ is zero except the $n, n$ entry, and similarly only the $n$-th component of the vector $F$ is nonzero:

$$
G_{n n}=\alpha R^{2}, \quad F_{n}=\alpha R^{2} T_{s} .
$$

This scheme is $O\left(h^{2}\right)$ in the sense that there is a constant $C>0$ (depending on the true solution $u$ ) such that

$$
\left\|u(\cdot, t)-u_{h}(\cdot, t)\right\| \leq C h^{2} \quad \text { for all } t \geq 0,
$$

\footnotetext{
${ }^{4}$ The terminology comes from mechanics, the discipline that popularized finite element methods.
} 
where

$$
\left\|u(\cdot, t)-u_{h}(\cdot, t)\right\|=\left[\int_{0}^{R}\left(u(r, t)-u_{h}(r, t)\right)^{2} r^{2} d r\right]^{1 / 2} .
$$

To obtain a fully discrete scheme, (39) is discretized in time by the CrankNicolson method,

$$
M\left(\frac{a^{(k+1)}-a^{(k)}}{\Delta t}\right)+(K+G)\left(\frac{a^{(k+1)}+a^{(k)}}{2}\right)=F,
$$

to obtain

$$
\left(M+\frac{\Delta t}{2} B\right) a^{(k+1)}=\left(M-\frac{\Delta t}{2} B\right) a^{(k)}+\Delta t F,
$$

where $a^{(k)}$ is the approximation to $a\left(t_{k}\right), t_{k}=k \Delta t, k=0,1,2, \ldots$.

We write $u^{(k)}(r)$ for the approximation to $u_{h}\left(r, t_{k}\right)$ obtained by estimating $a\left(t_{k}\right)$ by $a^{(k)}$. Then there exists constants $C_{1}, C_{2}>0$ such that, for all $k$,

$$
\left\|u\left(\cdot, t_{k}\right)-u^{(k)}\right\| \leq C_{1} h^{2}+C_{2} \Delta t^{2} .
$$

This error bound (and the earlier bound on the error in the semidiscrete solution) can be obtain by a straightforward generalization of the standard error analysis found in Thomée [2006].

\section{Examples}

We will now show several examples, demonstrating the effectiveness of the above analysis. In these examples, we compare the solution (2) of Newton's law of heating with an accurate solution of the heat equation computed by the finite element method described above.

Example 1 (A small iron ball). We first consider an iron ball approximately the size of a baseball: $R=3.7 \mathrm{~cm}$. The physical constants describing iron are $c=$ $0.437 \mathrm{~J} / \mathrm{g} \mathrm{K}, \rho=7.88 \mathrm{~g} / \mathrm{cm}^{3}$, and $\kappa=0.802 \mathrm{~J} / \mathrm{s} \mathrm{cm}$. Various references suggest values of $\alpha$ (the convection heat transfer coefficient in air) from $10^{-2}$ to $10^{-3}$ $\mathrm{W} / \mathrm{cm}^{2} \mathrm{~K}$; we will use a value of $\alpha=0.0045$. The corresponding value of $\bar{\alpha}$ is

$$
\bar{\alpha}=\frac{3 \alpha}{\rho c R} \approx 0.0010596 \text {. }
$$

We assume that the initial temperature of the ball is $T_{0}=0^{\circ} \mathrm{C}$ and that the temperature of the surrounding air is $T_{s}=25^{\circ} \mathrm{C}$, and simulate the temperature in the ball for one hour. The average temperature $\bar{u}$ computed by solving the heat equation and the temperature $T$ predicted by Newton's law of heating are indistinguishable on a graph (see Figure 2); the maximum difference between the two is about 0.038187 . 

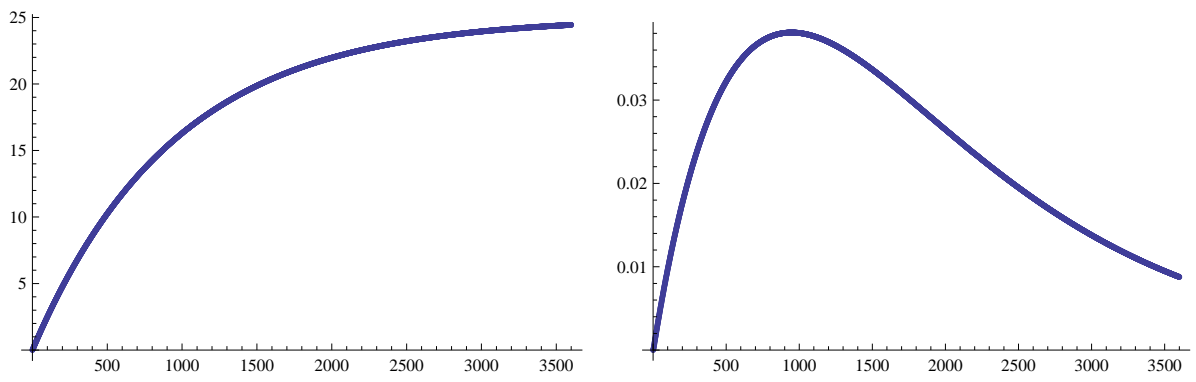

Figure 2. Left: The average temperature of the iron ball in Example 1. Right: The difference $\bar{u}(t)-T(t)$ between the temperatures calculated from the heat equation and Newton's law of heating. In both graphs, the horizontal axis is time in seconds, and the vertical is degrees Celsius.

In this example, we have $\beta=\frac{\alpha R}{\kappa} \approx 0.020761$, and the first-order bound on the error is

$$
\frac{\left|T_{s}-T_{0}\right|}{5 e} \beta \approx 0.038107 \text {. }
$$

With a small value of $\beta$, the analysis suggests that Newton's law is an accurate substitute for the heat equation, and that conclusion is confirmed by the numerical results. Moreover, the first-order bound on the difference between the two solutions is an excellent estimate of the actual difference.

Example 2 (A large iron ball). The second example is the same as the first, except now the radius of the ball is $R=100 \mathrm{~cm}$. The value of $\beta$ is now approximately 0.56110 , so we do not expect that Newton's law will yield a particularly accurate estimate of the true average temperature. We simulate the temperature for 20 hours (since it takes a long time to appreciably change the temperature in such a large ball).

As Figure 3 shows, the maximum difference between the two solutions is about 0.97333. The first-order bound on the error is

$$
\frac{\left|T_{s}-T_{0}\right|}{5 e} \beta \approx 1.0321 \text {. }
$$

Once again, the analysis proves to be quite accurate.

Example 3 (A small styrofoam ball). In the last example, Newton's law of heating, while not a bad approximation, did not accurately model the average temperature in the ball because the ball was so large. In this example, we consider the other reason why Newton's law might not work particularly well, namely, that heat flows slowly through the object compared to how quickly it flows from the surroundings to the object. We consider a styrofoam ball of radius $R=3.7 \mathrm{~cm}$. The physical 

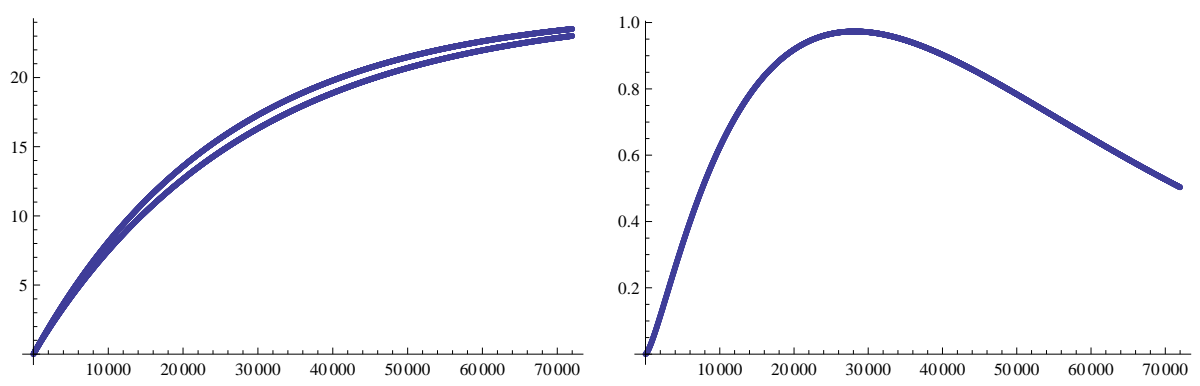

Figure 3. Left: The temperatures of the iron ball in Example 2, as predicted by the heat equation and Newton's law of heating. (The larger temperature is predicted by Newton's law.) Right: The difference $\bar{u}(t)-T(t)$ between the temperatures calculated from the heat equation and Newton's law.

parameters describing styrofoam are $c=0.209 \mathrm{~J} / \mathrm{g} \mathrm{K}, \rho=0.1 \mathrm{~g} / \mathrm{cm}^{3}$, and $\kappa=$ $3.3 \cdot 10^{-4} \mathrm{~J} / \mathrm{s} \mathrm{cm}$. We continue to use $\alpha=0.0045$, so now $\beta \approx 50.455$. Since $\beta \gg 1$, we expect Newton's law to yield a poor approximation to the true average temperature. This is confirmed in Figure 4, which shows a maximum error of about 14.363. (Since $\beta$ is so large, we should not expect the first-order error bound to be a good approximation to the actual error, and indeed it is not; the bound is about 92.806.) The value of $\bar{\alpha}$ is

$$
\bar{\alpha} \approx 0.17458 \text {. }
$$

A natural question arises in regard to this example: The results show that Newton's law does not produce good results with $\bar{\alpha}=3 \alpha /(\rho c R)$, but what if we use a
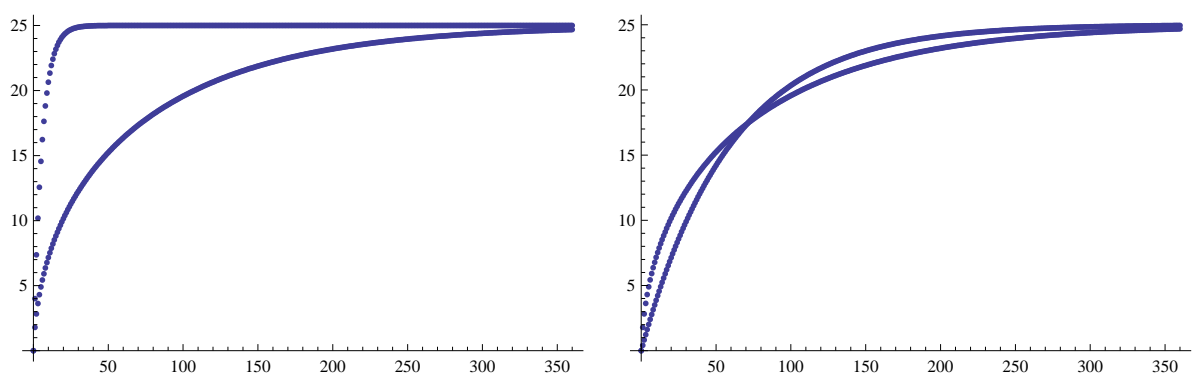

Figure 4. Left: The temperatures of the styrofoam ball in Example 3, as predicted by the heat equation and Newton's law of heating. (The larger temperature is predicted by Newton's law.) Right: The temperatures of the styrofoam ball in Example 3, as predicted by the heat equation and Newton's law with a better value of $\bar{\alpha}$. (The temperature predicted by Newton's law is eventually larger.) 
different value of $\bar{\alpha}$ ? To answer this question, we found the value of $\bar{\alpha}$ that produces a solution (2) as close as possible to $\bar{u}$ in the least-squares sense; that is, we found $\bar{\alpha}$ to minimize

$$
J(\bar{\alpha})=\sum_{k=1}^{N}\left(\bar{u}\left(t_{k}\right)-T_{s}+\left(T_{s}-T_{0}\right) e^{-\bar{\alpha} t_{k}}\right)^{2}
$$

(where $N$ is the number of time steps in the finite element simulation). We denote the optimal value of $\bar{\alpha}$ by $\tilde{\alpha}$; the result in this example is

$$
\tilde{\alpha} \approx 0.016805 \text {. }
$$

With this value of $\bar{\alpha}$, Newton's law yields a much improved estimate of the average temperature of the ball. Nevertheless, the result is still not very good (see Figure 4), which shows that, for this example, the true average temperature in the styrofoam ball is simply not well modeled by Newton's law of heating.

\section{Concluding remarks}

Our results show that for a small spherical object with the property that heat flows through the object more quickly than it flows to the surroundings $(\beta=\alpha R / \kappa \ll 1)$, Newton's law of heating provides a satisfactory model of the average temperature of the object. Moreover, the value

$$
\bar{\alpha}=\frac{3 \alpha}{\rho c R}
$$

is a satisfactory constant of proportionality in Newton's law. To carry out this analysis, we have assumed that the initial temperature of the object is constant throughout, and also that the temperature of the surroundings is held constant.

The alert reader may have noticed that the analysis suggests an even better value of $\bar{\alpha}$. The estimate of $\lambda_{1}$ in (30) suggests that

$$
\bar{\alpha}=\frac{3 \alpha}{\rho c R}\left(1-\frac{1}{5} \beta\right)
$$

would be an improved estimate of $\lambda_{1} /(\rho c)$ and hence lead to a better estimate of $\bar{u}$ by $T$. Indeed, the reader can easily check that this value of $\bar{\alpha}$ leads to an $O\left(\beta^{2}\right)$ bound for $|\bar{u}(t)-T(t)|, t \geq 0$.

Many textbook problems on Newton's law of cooling refer to inhomogeneous objects; perhaps the classic example is the cooling of a cup of coffee; see, for example, [Boyce and DiPrima 1992, Section 2.5, problem 14]. (Another popular example is the cooling of a corpse!) Nothing in our analysis allows us to address either inhomogeneities in the object or complex geometries. However, we can apply finite element simulation to an inhomogeneous sphere. For example, we can consider a hollow styrofoam ball filled with water, the closest we can get to 


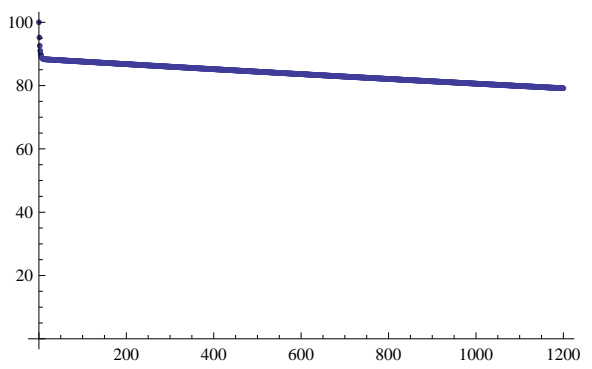

Figure 5. The average temperature in a hollow styrofoam ball filled with water, as computed by finite element simulation.

a coffee cup with our current work. We set the outer radius of the ball to $4.4 \mathrm{~cm}$ and the inner radius to $3.9 \mathrm{~cm}$ (so that it holds about $250 \mathrm{ml}$ of water), and assume that the initial temperature in both the water and the 'cup' is $T_{0}=100^{\circ} \mathrm{C}$. Finite element simulation (for 20 minutes) produces the average temperature shown in Figure 5. The results show that the average temperature initially drops quite rapidly, after which it decreases at a more moderate rate. The initial decrease (see the first few seconds in Figure 5) is due to the styrofoam cup's initial loss of heat to the surroundings; since styrofoam has a very small volumetric heat capacity (that is, $c$ measured in $\mathrm{J} / \mathrm{cm}^{3} \mathrm{~K}$ ), a small loss of heat energy translates to a relatively large decrease in temperature in the styrofoam. Once this decrease of average temperature in the styrofoam is complete, the average temperature in the entire ball decreases in a rate well modeled by a function of the form (2) (as the authors have verified), and so Newton's law is a good model after the first few seconds.

A more realistic initial condition would have the temperature of the water at, say, $100^{\circ} \mathrm{C}$ and the temperature of the styrofoam at room temperature. In this case, the average temperature in the entire ball is less than $100^{\circ} \mathrm{C}$ and initially increases as the hot water heats the styrofoam. Thereafter, again, Newton's law provides an adequate model.

\section{Acknowledgments}

The authors thank the anonymous referees for their careful reading of the paper. Their suggestions improved the final version noticeably.

\section{References}

[Arfken and Weber 2005] G. B. Arfken and H. J. Weber, Mathematical methods for physicists, 6th ed., Elsevier, New York, 2005. Zbl 1066.00001

[Boyce and DiPrima 1992] W. E. Boyce and R. C. DiPrima, Elementary differential equations and boundary value problems, 5th ed., Wiley, New York, 1992. Zbl 0807.34002 
[Folland 1995] G. B. Folland, Introduction to partial differential equations, 2nd ed., Princeton University Press, Princeton, NJ, 1995. MR 96h:35001 Zbl 0841.35001

[Gockenbach 2002] M. S. Gockenbach, Partial differential equations: analytical and numerical methods, Soc. Ind. Appl. Math., Philadelphia, 2002. MR 2003m:35001

[Haberman 2004] R. Haberman, Applied partial differential equations with fourier series and boundary value problems, 4th ed., Prentice Hall, Upper Saddle River, NJ, 2004.

[Thomée 2006] V. Thomée, Galerkin finite element methods for parabolic problems, 2nd ed., Series in Computational Math. 25, Springer, Berlin, 2006. MR 2007b:65003 Zbl 1105.65102

[Trantor 1968] C. J. Trantor, Bessel functions with some physical applications, Hart, New York, 1968.

[Watson 1944] G. N. Watson, A treatise on the theory of Bessel functions, 2nd ed., Cambridge University Press, Cambridge, 1944. Reprinted 1995. MR 96i:33010 Zbl 0849.33001

[Zill 2005] D. G. Zill, A first course in differential equations, 8th ed., Brooks/Cole, Belmont, 2005. Zbl 0785.34002

Received: $2008-11-25$

Revised: 2009-06-15 Accepted: 2009-07-13

msgocken@mtu.edu

Department of Mathematical Sciences, Michigan Technological University, 1400 Townsend Drive, Houghton, MI 49931-1295, United States

kkschmid@mtu.edu

Department of Mathematical Sciences, Michigan Technological University, 1400 Townsend Drive, Houghton, MI 49931-1295, United States 